\title{
Molecular Identification and Characterization of Bacillus Antagonist to Inhibit aflatoxigenic Aspergillus flavus
}

\author{
A.A. Bharose, H.P. Gajera*, Darshna G. Hirpara, V.H. Kachhadia and B.A. Golakiya \\ Department of Biotechnology, College of Agriculture, Junagadh Agricultural University, \\ Junagadh, 362001, Gujarat, India \\ *Corresponding author
}

\section{A B S T R A C T}

The knowledge concerning the behavior of these Bacilli as antagonists and genetic analysis is essential for their effective use and the commercialization. The present study was focused on the analysis of the genetic diversity of rhizobacterial isolates of Bacillus

Keywords

A. flavus,

Aflatoxigenic,

Bacillus,

Antagonism,

Molecular diversity, 16S rRNA gene.

Article Info

Accepted:

20 February 2017

Available Online: 10 March 2017 using PCR based RAPD technique and selection of best biocontrol antifungal Bacillus strain with aflatoxin producing Aspergillus by antagonism on PDA medium. About 16 different strains of bacteria were isolated from healthy and infested rhizosphere of groundnut using $\mathrm{N}$-agar medium. The isolates were identified based on morphological and microscopic characters such as colony color, shape, size, margin, opacity, texture, elevation, pigmentations, Gram staining and spore staining. Bacterial isolate JND-KHGn29-A and JND-KSGn-30-L were recorded to be a best antagonist as of its ability to inhibit most toxic fungus $A$. flavus JAM-JKB-BHA-GG20 (58.20\%) after screening with 16 Bacillus isolates. The best antagonist bacterial isolate JND-KHGn-29-A also evidenced with nitrate reduction and sederophore as PGPR activity. The genetic diversity was studied among bacterial 16 bacterial isolates by using RAPD markers. Out of 38 primer, total 10 primers showed amplification. The highest numbers of 19 bands were produced by OPA07 primer and lowest 1 band was produced by OPJ-07. The similarity index values generated by Jaccard's similarity coefficient and dendrogram grouped all bacterial isolates into two main clusters at $61 \%$ similarity. The best and least bacterial antagonist were grouped into different clusters depicting genetically difference between isolates. The $16 \mathrm{~S}$ rDNA study revealed that the best and least antagonist bacterial isolates JND-KHGn-29A and JND-KHGn-29B were identified as Bacillus subtilis.

\section{Introduction}

The rhizosphere is a complex system in which beneficial plant microbe interactions play vital role in agriculture to sustain the plant growth and productivity. Plant growth promoting rhizobacteria (PGPR) exert the positive effect on plant growth through various mechanisms either directly or indirectly (Joseph et al., 2007). The bacillus bacteria play vital role in plant health by direct and indirect activities. The direct activity attributed by increased uptake of nitrogen (Kennedy et al., 2004) phytohormones synthesis (Hayat et al. 2008 a, b) solubilization of phosphorus and siderophore production (Pidello, 2003) while indirect activity include realise of phytoharmones like secondary metabolites viz. HCN, ammonia, antibiotics, and volatile metabolites (Owen 
and Zlor, 2001). A large number of researchers have reported significant increases in productivity of important agronomic crops by inoculation with PGPR (Bashan et al., 2004). The ability of the antagonistic rhizobacteria is highly influenced by their morphological characteristics to inhibit the pathogens.

RAPD-PCR technique has been proposed as a tool for generating taxon-specific markers with different specificities (Kim et al., 2007). RAPD-PCR analyses have been shown to be suitable for generating strain and speciesspecific amplification profiles (Ronimus et al., 1997). Jeyaram et al. (2008) used RAPDPCR analyses for confirming $82 \mathrm{~B}$. subtilis strains from Hawaijar, a traditional Indian fermented soy food. Torriani et al. (2001) used RAPD-PCR for species differentiation among similar Lactobacillus plantarum, $L$. pentosus and L. paraplantarum. DNA-based identification methods such as 16S rRNA gene sequencing have been used widely for the purpose of identification and typing of microorganisms isolated from natural environments including fermented foods (Levine et al., 2005).

The cultivated groundnut (Arachis hypogaea L.) is the most important oilseed crop and its kernels are also eaten raw, boiled or roasted. After the crop harvest, haulm and the expeller oil cake is used for animal feed. Aflatoxin contamination in groundnut seed is a major problem affecting the export. Aflatoxin contamination of the seed by $A$. flavus can occur during pre-harvest, during harvest and drying in the field, and during transportation and storage. The objectives of the present study was to evaluate the best bacterial biocontrol agent using in vitro antagonism against toxinogenic $A$. flavus and study the molecular diversity, microbial identification using $16 \mathrm{~S}$ rRNA of the antagonist isolated from healthy and infested rhizosphere of groundnut. Furthermore, to confirm their plant growth promoting activities for the conventional use of commonly applied fertilizers and pesticides.

\section{Materials and Methods}

The present study was conducted to isolate native strains of rhizobacteria from healthy and infested rhizosphere of groundnut.

\section{Collection of soil samples and isolation of rhizospheric bacteria}

Rhizosphere soil was collected from groundnut fields healthy and infested with fungal disease like stem rot, color rot etc. Soil samples were collected from 16 rhizospheric soils of different field crops. For the isolation of native rhizobacteria $1 \mathrm{~g}$ of soil was suspended in $90 \mathrm{ml}$ distilled autoclaved water. Serial dilution agar plate method was used for further processing of the prepared soil suspension, Suitable dilutions were plated on $\mathrm{N}$-agar media. All the plates were incubated for 2 days at $28^{\circ} \mathrm{C}$ (Aneja, 2002). Well isolated pure bacterial colony were selected and transferred on freshly prepared $\mathrm{N}$-agar media and stored at low temperature in refrigerator till further use (Alemu, 2013).

\section{Morphological characteristics of bacterial isolates}

Morphological characteristics of the colony of each isolate were examined on the NA-agar plates after incubated for 3 days at $28^{0} \mathrm{C}$. Then colony characterization of $\mathrm{N}$-agar media was carried out viz., size, shape, margin, elevation, texture, opacity and pigmentation.

\section{Microscopic examination of bacterial isolates}

Standard microbiological methods were used to fix the cells to slides for Gram staining and 
observed under Zeiss Axiocam Imager, model $\mathrm{Z} 2$. Endospore staining was carried out by the method of Aneja (2003).

In vitro antagonism of bacterial isolates against aflatoxinogenic $A$. flavus

To derive best biocontroller, all bacterial isolates were subjected to in vitro antagonism with highly virulent and aflatoxigenic Aspergillus strain. The most responsive fungal isolate was cultivated in petriplate with $20 \mathrm{ml}$ of Potato Dextrose Agar for seven days. Discs of $5 \mathrm{~mm}$ diameter were cut and removed from the growing borders of the colonies and transferred to another petriplates with Potato Dextrose Agar. Aflatoxicity of isolated pathogen was tested using biochemical method. In this method, the reverse side of colonies of toxin producing strains on potato dextrose agar (PDA) medium turns from yellow to pink immediately after exposure to ammonium hydroxide vapor. The test fungus was placed in the each center of the petriplate and approximately $3 \mathrm{~cm}$ away bacterial isolates. The bacterial isolates were spread in round shape around the bid of the fungus. Control plates were maintained only with pathogen. All the inoculated plates were incubated at 28 $\pm 2^{0} \mathrm{C}$ temperature and observed after ten days for growth of antagonist bacteria and test fungus (Reddy et al., 2008). The experiment was conducted in completely randomized design with three replications. At the end of incubation period, radial growth of pathogen A. flavus was measured and Index of antagonism was determined by following the method of Zarrin (2009) as depicted below

$\%$ Growth Inhibition $=\mathrm{C}-\mathrm{T} / \mathrm{C} * 100$

Where, $\mathrm{C}=$ colony diameter of pathogen in control $\mathrm{T}=$ colony diameter pathogen in inhibition plate
Defense related and plant growth promoting (PGPR) activity of bacterial isolates

Bacterial isolates were grown in $250 \mathrm{ml}$ conical flasks containing $100 \mathrm{ml}$ of LB broth for $48 \mathrm{~h}$ on a rotary shaker at $28{ }^{\circ} \mathrm{C}$. Cells were taken by centrifugation at $10,000 \mathrm{~g}$ for $10 \mathrm{~min}$ at $4^{\circ} \mathrm{C}$. The pellet was re-suspended in $100 \mathrm{ml}$ of sterile distilled water (density measured as 1 at $600 \lambda$ ).

\section{Siderophore production}

Siderophore production was assayed by spot inoculation of bacterial isolates in the CAS agar medium (Clark and Bavoil, 1994). The plates were incubated at $28^{\circ} \mathrm{C}$ for 5 days. Siderophore production was observed by the development of orange halo around the colonies.

\section{Indole acetic acid (IAA) production}

The bacterial isolates were inoculated for determination of IAA like substances in 100 $\mathrm{ml}$ of $\mathrm{N}$ broth supplemented with tryptophan $0.1 \mathrm{mg} \cdot \mathrm{ml}^{-1}$. The cultures were incubated at $28 \pm 2^{\circ} \mathrm{C}$ for 3 days $(72 \mathrm{hr}$ ) with occasional shaking. After incubation, the cultures were centrifuged at $10,000 \mathrm{rpm}$ for $10 \mathrm{~min}$. Two millilitres of freshly prepared Salkowski's reagent $\left(1 \mathrm{ml}\right.$ of $0.5 \mathrm{M} \mathrm{FeCl}_{3}$ in $50 \mathrm{ml}$ of $35 \%$ $\mathrm{HClO}_{4}$ ) was added to $1 \mathrm{ml}$ of culture supernatant. The reaction mixture was incubated at $30^{\circ} \mathrm{C}$ for $30 \mathrm{~min}$. Development of pink colour indicates the production of IAA (Aneja, 2003).

\section{Phosphate solubilisation}

Phosphate solubilization test of isolated bacterial isolates was carried out as described by Ravikumar (2002). The plates were prepared with Pikovskaya's medium. The isolates were streaked on the plates and 
incubated in an incubator at $28^{\circ} \mathrm{C}$ for 7 days. The plates were observed for the clear zone around (Light bluish) the colonies and consider positive for phosphate solublizing activity.

\section{Nitrate reduction}

The bacterial isolates were checked for nitrate reduction. The medium containing beef extract (3.0gm), geletin peptone (5.0gm), KNO3 (1.0gm) and deionised water $(1000 \mathrm{ml})$ was prepared and heated gently. Then, $20 \mathrm{ml}$ broth was taken in sugar tubes and Durham's tube was added inverted and autoclaved. After autoclaving each tube were heavily inoculated and incubated for $48 \mathrm{hrs}$. Two drops of reagent $\mathrm{A}(\mathrm{N}, \mathrm{N}$ - dimethylphenolpthalamine $(0.6 \mathrm{ml})$ and $5 \mathrm{~N}$ acetic acid $(100 \mathrm{ml})$ and reagent $\mathrm{B}$ (sulphanilic acid-0.8gm) and acetic acid $(100 \mathrm{ml})$ were added in one test tube and then $1 \mathrm{ml}$ broth was added in it. The positive test was confirmed by appearance of red color within two minutes where as negative test was confirmed by adding zinc dust for visualizing red color in same tubes.

\section{Molecular characterization of antagonist bacterial isolates}

\section{Isolation of genomic DNA}

The genomic DNA was isolated from overnight culture in nutrient broth by the method of Martinez et al. (2002). Cells were recovered by centrifugation at $13,000 \times \mathrm{g}$ for 3 min. Cell pellet was resuspended in $1 \mathrm{ml}$ of $10 \mathrm{mM}$ Tris- $\mathrm{HCl}, \mathrm{pH}$ 8.0, 10 mM EDTA, 100 $\mathrm{mM} \mathrm{NaCl}, 2 \%(\mathrm{w} / \mathrm{v})$ SDS, and $400 \mu \mathrm{g} / \mathrm{ml}$ proteinase $\mathrm{K}(20 \mathrm{mg} / \mathrm{ml})$ and incubated for 30 min at $55{ }^{\circ} \mathrm{C}$. Total DNA was isolated using method described by Amer et al. (2011). The aqueous upper layer was transferred into a fresh tube and same volume of isopropanol was added. DNA was precipitated by centrifugation at $13,000 \times \mathrm{g}$ for $20 \mathrm{~min}$ at $4{ }^{\circ} \mathrm{C}$ followed by washing with $70 \%(\mathrm{v} / \mathrm{v})$ ethanol, dried under vacuum, and resuspended in $50 \mu \mathrm{l}$ sterile water.

\section{RAPD-PCR analysis}

RAPD-PCR assays were performed in $15 \mu 1$ reaction volume and each tube contained Taq DNA polymerase, $10 \mathrm{pmol}$ primers, and $1 \mu \mathrm{g}$ of template DNA. PCR was done using Thermal cycler (Veriti, Model 96 well thermo cycler) and amplification conditions included an initial denaturation step at $94{ }^{\circ} \mathrm{C}$ for $4 \mathrm{~min}$, 35 cycles of $94{ }^{\circ} \mathrm{C}$ for $1 \mathrm{~min} ., 36{ }^{\circ} \mathrm{C}$ for 15 $1 \mathrm{~min} ., 72^{\circ} \mathrm{C}$ for $2 \mathrm{~min}$, and final extension at $72{ }^{\circ} \mathrm{C}$ for 10 min (Archana et al., 2007). RAPD-PCR products were analyzed by agarose gel $(1.5 \%)$ electrophoresis with a molecular size marker (1 kb DNA ladder). DNA bands were visualized under UV light and banding patterns of amplified DNA was scored as present or absent in binary matrix. The RAPD data were subjected to statistical analysis for the calculation of Jaccard's similarity coefficient and cluster analysis by UPGMA (unweighted pair-group method with arithmetic averages) using NTSYSpc2.02i software.

\section{PCR amplification of $B$. subtilis species- specific 16S rRNA}

Species-specific primer set for $B$. subtilis Bsub5F (5'- AAGTCGAGCGGACAGATG G-3') and Bsub 3R (5'- CCAGTTTCCAATGACCCT CCCC -3') were used. PCR was performed using a Thermal cycler (Veriti, Model 96 well thermo cycler). The reaction mixture $(50 \mu \mathrm{l})$ contained $1 \mu \mathrm{g}$ of template DNA, $1 \mu \mathrm{l}$ of each primer $(10 \mathrm{pmol}), 5 \mu \mathrm{l}$ of dNTP $(0.25 \mathrm{mM})$, and $0.5 \mu \mathrm{l}$ of Taq DNA polymerase. The following thermal cycling conditions were used: initial denaturation step at $94{ }^{\circ} \mathrm{C}$ for 2 min and 30 cycles consisting of denaturation at $94{ }^{\circ} \mathrm{C}$ for $15 \mathrm{~s}$, annealing at $55^{\circ} \mathrm{C}$ for 20 min and primer extension at $72{ }^{\circ} \mathrm{C}$ for $1 \mathrm{~min}$. 
PCR products were analyzed by agarose gel (2\%) electrophoresis with a molecular size maker (100 bp DNA ladder). Bacterial isolates were identified based on $16 \mathrm{~S}$ rDNA sequencing using MicroSeq ${ }^{\circledR 500 ~ 16 S ~ r D N A ~}$ bacterial identification kits (PN 4346298) as per manufacture protocol, by using $3130 \mathrm{XL}$ gene sequencer. The $16 \mathrm{~S}$ rDNA gene was amplified by using PCR and sequencing kit supplied by Invitrogen Pvt. Ltd., USA. The PCR and sequencing reaction were carried out as per the protocol described in the above said kit. Therefore, amplified amplicon from bacillus specific primer set were further taken for sequencing. The obtained sequences were BLAST on NCBI data base.

Data were statistically analyzed by analysis of variance technique and comparison among means was made by completely randomized design (CRD) for study in the significance of various data (Fisher and Yates, 1948).

\section{Results and Discussion}

\section{Morphological characteristics of bacterial isolates}

Total 16 different strains of bacteria were isolated from healthy and infested rhizosphere of groundnut and colony color, shape, size, margin, opacity, texture, elevation and pigmentations of all sixteen isolates were determined by observing the plates after 7 days on $\mathrm{N}$ agar medium (Table 1 ).

\section{In vitro antagonism of bacterial isolates with virulent Aspergillus biocontrol agent}

All the bacterial isolates were screened with JAM-JKB-BHA-GG20 most toxic isolate of Aspergillus flavus fungus. Growth inhibition of Aspergillus flavus during in vitro interaction with biocontrol bacterial agents were recorded at 7 DAI (Table 2). The antagonist result depicted that the bacterial isolate T1 (JND-KHGn-29-A was the best antagonist inhibiting highest growth (58.20 $\%$ ) of test pathogen A. flavus followed by isolate T15 (JND-KSCa-22) (48.04\%), T16 (JND-KSCa-23) (45.30 \%) and T4 (JNDKSGn-30-B) (47.80\%). Whereas, bacterial isolate T2 (JND-KHGn-29-B) (0.00 \%) evidenced as least antagonist among 16 bacterial isolates followed by isolates T6 (JND-KSGn-30-D), T8 (JND-KSGn-30-F), T3 (JND-KSGn-30-A), T12 (JND-KSGn-30J) and T10 (JND-KSGn-30-H), against toxigenic A. flavus isolate JAM-JKB-BHAGG20 (Table 2, Fig. 1 and Fig. 2).

\section{Assay of defense related substances and PGPR activities}

All 16 different strains of bacteria, isolated from healthy and infested rhizosphere of groundnut. All the bacterial isolates were screened for gram's staining and their defense related substances (Table 5). The observations were recorded as presence (+) or absence (-) of defence related substances. All isolates act differently to defence related substances. The bacterial isolate JND-KHGn-29-A was found to have nitrate reduction and sederophore activity. The best antagonist bacterium was identified as Bacillus after its colony characterization by gram's staining $(+v e)$ and spore forming.

PGPRs bear inhibitory effects for various pathogens on plant growth and development in the forms of biocontrol agents. The PGPR activities vary with the bacterial species and also with the physico-chemical conditions of the rhizosphere. (Glick and Bernard, 2012). Biocontrol of plant diseases, especially of fungal origin, has been achieved using microorganisms Pseudomonas sp. nd Bacillus sp (Ligon et al., 2000). Raaijmakers et al. (2002) examined IAA production by test isolates of Bacillus spp. The results were contradictory with our results that, best antagonist bacterial isolate JND-KHGn-29-A showed negative IAA test and least antagonist 
Bacillus JND-KHGn-29-B bacterial isolate showed positive IAA test.

In present study best antagonist Bacillus isolate JND-KHGn-29-A (isolate no. 1) was found to have better nitrate reduction activity. Similar nitrate reductases activity of Bacillus was reported by Nakano et al. (1998). B. subtilis can use nitrite or nitrate as a terminal acceptor of electrons. Production of siderophore by best antagonist Bacillus isolate JND-KHGn-29-A was observed. The production of siderophore by rhizobacteria has been confirmed by previous studies (Noori and Saud, 2012). A direct correlation was found to exist between siderophore production and antifungal activity (Raval and Desai, 2012). The sederophore create iron limiting conditions for pathogenic fungus and prevents it from invading and colonizing the plant roots (Meyer and Stintzi, 1998). The similar results were also obtained in the present study corresponding to siderophore production with greater antagonistic activity of the Bacillus isolate JND-KHGn-29-A and JND-KSGn-30-L.

\section{Molecular diversity of bacterial isolates using RAPD}

The polymorphisms can be detected by the use of random amplified polymorphic DNA (RAPD) which does not require prior knowledge of the genome. The RAPD has been commonly used for fingerprinting of biocontrol agents Chapon et al. (2002). In the present investigation, amplified products were observed when the genomic DNA of bacterial isolates was subjected to RAPD analysis using 38 random decamer primers.

Initially total 38 primers were screen for polymorphism using genomic DNA of isolates. Out of total 38 primer total 10 primer gave amplification which were further selected for amplification of genomic DNA of 16 bacterial isolates. The highest numbers of
19 bands were produced by OPA-07 primer followed by 15 bands of OPK-03 primer. The lowest 1 bands were produced by OPJ-07. The largest fragment of $3798 \mathrm{bp}$ and the smallest fragment of $116 \mathrm{bp}$ were amplified by OPH-15 primer (Table 6). The polymorphism information content (PIC) was calculated for each primer and it was varied between 0.84 (OPD-03) and 1.00 (OPA-07, OPA-18, OPH-15, OPJ-07, OPK-03, OPG-08, B1, OPO-06 and OPD-03) with an average of 0.95 per primer. The details of polymorphism pattern of individual primer are given in (Table 4).

\section{Cluster analysis of RAPD}

The similarity index values generated by Jaccard's similarity coefficient among 16 bacterial isolates based on RAPD data showed the similarity coefficient ranging from 0.5446 to 0.8911 (54.46 \% to $89.11 \%$ ). The more genetic similarity $(89.11 \%)$ was observed between isolate 12 (JND-KSGn-30$\mathrm{J})$ and isolate 7 (JND-KSGn-30-E) followed by $(86 \%)$ between isolate 10 (JND-KSGn-30$\mathrm{H}$ ) and isolate 9 (JND-KSGn-30-G), whereas lowest genetic similarity $(54.46 \%)$ was observed between isolate 2 (JND-KHGn-29B) and isolate 1 (JND-KHGn-29-A), isolate 9 (JND-KSGn-30-G) and isolate 1 (JNDKHGn-29-A) and isolate 9 (JND-KSGn-30G) and isolate 2 (JND-KHGn-29-B) followed by $55 \%$ genetic similarity between isolates 14 (JND-KSGn-30-L) and isolate 8(JND-KSGn30-F).

The similarity index values generated by Jaccard's similarity coefficient were used to construct dendrogram using UPGMA method was depicted in Fig. 3. The dendrogram grouped all bacterial isolates into two main clusters at $61 \%$ similarity viz. cluster I and cluster II. Cluster I was again sub divided into cluster IA and cluster IB at $61.8 \%$ similarity (Fig. 3). 
Table.1 Morphological characterization of bacterial isolates collected from groundnut rhizosphere

\begin{tabular}{|c|c|c|c|c|c|c|c|c|c|c|}
\hline Crop name & $\begin{array}{l}\text { Rhizospher } \\
\text { e Condition }\end{array}$ & Code Name & $\begin{array}{l}\text { Colony } \\
\text { Shape }\end{array}$ & Size & Color & Margin & Opacity & $\begin{array}{l}\text { Texture/ } \\
\text { Consistenc } \\
\mathbf{y}\end{array}$ & $\begin{array}{l}\text { Elevatio } \\
\text { n }\end{array}$ & $\begin{array}{l}\text { Pigmentatio } \\
\text { n }\end{array}$ \\
\hline Ground nut & Healthy & JND-KHGn-29-A & irregular & medium & white & Undulate & opaque & brittle & flat & no \\
\hline Ground nut & Healthy & JND-KHGn-29-B & circular & tiny & white & Entire & opaque & dry & raised & no \\
\hline Ground nut & SICK & JND-KSGn-30-A & circular & tiny & $\begin{array}{l}\text { yellowis } \\
\mathrm{h}\end{array}$ & Entire & opaque & dry & raised & no \\
\hline Ground nut & SICK & JND-KSGn-30-B & irregular & medium & white & Undulate & opaque & brittle & flat & red \\
\hline Ground nut & SICK & JND-KSGn-30-C & irregular & small & white & Curled & opaque & dry & umbonate & no \\
\hline Ground nut & SICK & JND-KSGn-30-D & filamentous & large & white & Filiform & opaque & dry & flat & no \\
\hline Ground nut & SICK & JND-KSGn-30-E & circular & small & white & Entire & opaque & moist & raised & no \\
\hline Ground nut & SICK & JND-KSGn-30-F & irregular & large & white & Curled & opaque & dry & umbonate & red \\
\hline Ground nut & SICK & JND-KSGn-30-G & circular & tiny & white & Entire & opaque & moist & umbonate & no \\
\hline Ground nut & SICK & JND-KSGn-30-H & irregular & large & white & Undulate & opaque & dry & flat & red \\
\hline Ground nut & SICK & JND-KSGn-30-I & irregular & medium & white & Undulate & opaque & brittle & flat & no \\
\hline Ground nut & SICK & JND-KSGn-30-J & irregular & large & yellow & Curled & opaque & dry & umbonate & yellow \\
\hline Ground nut & SICK & JND-KSGn-30-K & irregular & large & white & Undulate & opaque & buttery & raised & red \\
\hline Ground nut & SICK & JND-KSGn-30-L & irregular & large & white & Undulate & opaque & brittle & flat & cream \\
\hline Castor & SICK & JND-KSCa-22 & circular & small & white & Entire & opaque & viscous & convex & no \\
\hline Castor & SICK & JND-KSCa-23 & circular & small & white & Entire & opaque & viscous & convex & no \\
\hline
\end{tabular}


Table.2 Percent growth inhibition of A. flavus by Bacterial antagonists

\begin{tabular}{|c|l|c|}
\hline $\begin{array}{c}\text { Isolate } \\
\text { No. }\end{array}$ & \multicolumn{1}{|c|}{ Treatment } & $\begin{array}{c}\text { \% Growth } \\
\text { Inhibition } \\
\text { 7 DAI }\end{array}$ \\
\hline T1 & JND-KHGn-29-A X Pathogen-AFvs* & 58.20 \\
\hline T2 & JND-KHGn-29-B X Pathogen -AFvs & 0.00 \\
\hline T3 & JND-KSGn-30-A X Pathogen-AFvs & 6.04 \\
\hline T4 & JND-KSGn-30-B X Pathogen-AFvs & 47.80 \\
\hline T5 & JND-KSGn-30-C X Pathogen-AFvs & 25.82 \\
\hline T6 & JND-KSGn-30-D X Pathogen-AFvs & 2.20 \\
\hline T7 & JND-KSGn-30-E X Pathogen-AFvs & 8.79 \\
\hline T8 & JND-KSGn-30-F X Pathogen-AFvs & 5.00 \\
\hline T9 & JND-KSGn-30-G X Pathogen-AFvs & 20.88 \\
\hline T10 & JND-KSGn-30-H X Pathogen-AFvs & 7.14 \\
\hline T11 & JND-KSGn-30-I X Pathogen-AFvs & 22.53 \\
\hline T12 & JND-KSGn-30-J X Pathogen-AFvs & 21.43 \\
\hline T13 & JND-KSGn-30-K X Pathogen-AFvs & 6.04 \\
\hline T14 & JND-KSGn-30-L X Pathogen-AFvs & 50.27 \\
\hline T15 & JND-KSCa-23 X Pathogen-AFvs & 48.04 \\
\hline T16 & JND-KSCa-22 X Pathogen-AFvs & 45.30 \\
\hline T17 & Control = Pathogen & 0.00 \\
\hline & S.Em. \pm & 0.444 \\
\hline & C.D. @ 5\% & 1.275 \\
\hline C.V. \% & 3.783 \\
\hline
\end{tabular}

* A. flavus JAM-JKB-BHA-GG20 (Isolate-3) - most toxic to produce aflatoxin 
Table.3 Characterization of bacterial isolates for PGPR activity

\begin{tabular}{|c|c|c|c|c|c|c|c|}
\hline $\begin{array}{c}\text { Isolate } \\
\text { No. }\end{array}$ & Bacterial Isolates & IAA & $\begin{array}{c}\text { Gram's } \\
\text { staining }\end{array}$ & PSB & $\begin{array}{c}\text { Spore- } \\
\text { staining }\end{array}$ & $\begin{array}{c}\text { Nitrate } \\
\text { reduction }\end{array}$ & Sederophore \\
\hline 1 & JND-KHGn-29-A & - & + & - & - & + & + \\
\hline 2 & JND-KHGn-29-B & + & - & - & - & - & - \\
\hline 3 & JND-KSGn-30-A & - & - & + & - & + & + \\
\hline 4 & JND-KSGn-30-B & + & + & - & - & - & \\
\hline 5 & JND-KSGn-30-C & - & - & + & - & + & + \\
\hline 6 & JND-KSGn-30-D & + & + & - & + & & - \\
\hline 7 & JND-KSGn-30-E & - & - & + & - & + & + \\
\hline 8 & JND-KSGn-30-F & + & + & - & - & - & - \\
\hline 9 & JND-KSGn-30-G & - & - & + & + & - & - \\
\hline 10 & JND-KSGn-30-H & - & + & - & - & - & - \\
\hline 11 & JND-KSGn-30-I & - & - & - & - & - & + \\
\hline 12 & JND-KSGn-30-J & - & - & - & + & - & + \\
\hline 13 & JND-KSGn-30-K & - & + & - & + & - & - \\
\hline 14 & JND-KSGn-30-L & - & - & - & - & - & + \\
\hline 15 & JND-KSCa-23 & + & - & - & - & - & - \\
\hline 16 & JND-KSCa-22 & - & - & + & - & - & - \\
\hline
\end{tabular}

Table.4 Polymorphism of 16 bacterial isolates generated with different RAPD primers

\begin{tabular}{|c|c|c|c|c|c|c|c|c|c|c|}
\hline \multirow[t]{2}{*}{$\begin{array}{l}\text { Sr. } \\
\text { No. }\end{array}$} & \multirow[t]{2}{*}{$\begin{array}{l}\text { RAPD } \\
\text { Primer }\end{array}$} & \multirow{2}{*}{$\begin{array}{l}\text { Bend } \\
\text { Size } \\
\text { (bp) }\end{array}$} & \multirow{2}{*}{$\begin{array}{c}\text { Total } \\
\text { No. of } \\
\text { Bends } \\
\text { (A) }\end{array}$} & \multicolumn{3}{|c|}{$\begin{array}{l}\text { Polymorphic Bands } \\
\text { (B) }\end{array}$} & \multirow{2}{*}{$\begin{array}{c}\text { Mono- } \\
\text { Mor } \\
\text { phic } \\
\text { Bend }\end{array}$} & \multirow{2}{*}{$\begin{array}{c}\% \\
\text { Poly- } \\
\text { Mor } \\
\text { Phism } \\
\text { (B/A) }\end{array}$} & \multirow[t]{2}{*}{ PIC* } & \multirow[t]{2}{*}{ RPI } \\
\hline & & & & $\mathbf{S}$ & $\mathbf{U}$ & $T$ & & & & \\
\hline 1 & OPA-07 & $233-1678$ & 19 & 14 & 5 & 19 & 0 & 100.00 & 1.00 & 19.00 \\
\hline 2 & OPA-18 & $199-2174$ & 14 & 12 & 2 & 14 & 0 & 100.00 & 1.00 & 13.98 \\
\hline 3 & OPH-15 & $116-3798$ & 7 & 7 & 0 & 7 & 0 & 100.00 & 0.89 & 6.22 \\
\hline 4 & OPJ-07 & 253 & 1 & 1 & 0 & 1 & 0 & 100.00 & 0.89 & 0.89 \\
\hline 5 & OPK-03 & $213-2335$ & 15 & 8 & 7 & 15 & 0 & 100.00 & 1.00 & 14.98 \\
\hline 6 & OPG-08 & $207-1428$ & 11 & 5 & 6 & 11 & 0 & 100.00 & 0.98 & 10.80 \\
\hline 7 & B1 & $138-1359$ & 12 & 12 & 0 & 12 & 0 & 100.00 & 0.99 & 11.88 \\
\hline 8 & OPO-06 & $240-1792$ & 10 & 8 & 2 & 10 & 0 & 100.00 & 0.94 & 9.38 \\
\hline 9 & OPC-13 & $178-1102$ & 8 & 7 & 0 & 7 & 1 & 87.50 & 0.99 & 7.91 \\
\hline 10 & OPD-03 & $345-1205$ & 3 & 3 & 0 & 3 & 0 & 100.00 & 0.84 & 2.53 \\
\hline \multicolumn{3}{|c|}{ Total } & 86.00 & 61.00 & 24.00 & 85.00 & 1 & 887.50 & 8.52 & 83.58 \\
\hline \multicolumn{3}{|c|}{ Average } & 10 & 7.3 & 2.6 & 9.44 & 0.11 & 98.61 & 0.95 & 9.29 \\
\hline
\end{tabular}

$\mathbf{S}=$ Shared; $\mathrm{U}=$ Unique; $\mathbf{T}=$ Total Polymorphic Bands;

$\mathbf{P I C}=$ Polymorphism Information Content; RPI = RAPD Primer Index 
Table.5 Unique RAPD markers associated with antagonistic bacterial isolates

\begin{tabular}{|c|c|c|c|c|c|c|c|c|c|c|c|c|c|c|c|c|}
\hline \multirow[b]{2}{*}{$\begin{array}{c}\text { RAPD } \\
\text { Primers }\end{array}$} & \multicolumn{16}{|c|}{ Bacterial Isolates } \\
\hline & 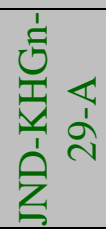 & 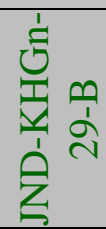 & 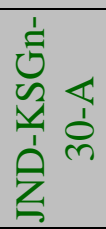 & 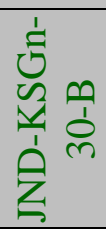 & 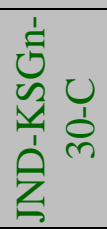 & 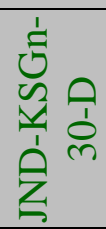 & 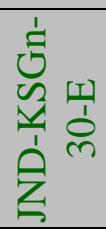 & $\begin{array}{l}\stackrel{1}{0} \\
\sqrt[n]{1} \\
\frac{1}{1} \\
\hat{y}^{1}\end{array}$ & 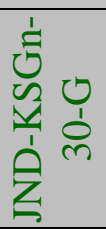 & 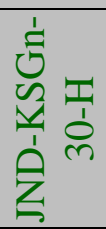 & 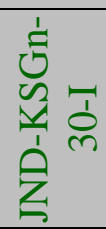 & $\begin{array}{l}\stackrel{1}{0} \\
\sqrt[n]{n} \\
\frac{1}{1} \\
\hat{y}^{1}\end{array}$ & 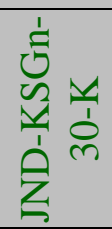 & 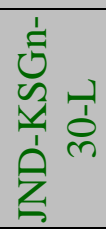 & 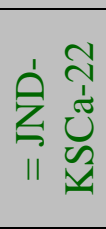 & 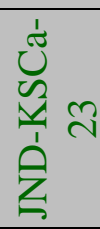 \\
\hline OPA-18 & 1950 & 1135 & - & - & - & - & - & - & - & - & - & - & - & - & - & - \\
\hline \multirow[t]{2}{*}{ OPA-07 } & 966 & 875 & - & - & 493 & - & - & - & - & - & - & - & - & - & - & - \\
\hline & 191 & - & - & - & 331 & - & - & - & - & - & - & - & - & - & - & - \\
\hline \multirow[t]{4}{*}{ OPK-03 } & 1642 & 305 & - & - & - & - & - & - & - & - & - & - & 2308 & - & - & - \\
\hline & 671 & - & - & - & - & - & & & & & & & 1050 & & & \\
\hline & 362 & - & - & - & - & - & - & - & - & - & - & - & - & - & - & - \\
\hline & 168 & - & - & - & - & - & - & - & - & - & - & - & - & - & - & - \\
\hline \multirow[t]{3}{*}{ OPG-08 } & 650 & 1196 & - & - & - & - & - & - & - & - & - & - & - & 1001 & - & 701 \\
\hline & - & 401 & - & - & - & - & - & - & - & - & - & - & - & - & - & - \\
\hline & - & 281 & - & - & - & - & - & - & - & - & - & - & - & - & - & - \\
\hline B1 & - & 1555 & - & - & - & - & - & - & - & - & - & - & 1792 & - & - & - \\
\hline $\begin{array}{c}\text { Total } \\
\text { No. }\end{array}$ & 8 & 7 & 0 & 0 & 2 & 0 & 0 & 0 & 0 & 0 & 0 & 0 & 3 & 1 & 0 & 1 \\
\hline
\end{tabular}


Table.6 Molecular identification of bacterial isolates $B$. subtilis using $16 \mathrm{~S}$ rRNA gene specific primers and their blast results

\begin{tabular}{|c|c|c|c|c|c|}
\hline $\begin{array}{l}\text { Sr. } \\
\text { No. }\end{array}$ & Isolate Code & $\begin{array}{c}\text { Amplification } \\
\text { product } \\
\text { (Fragment } \\
\text { size in bp) }\end{array}$ & $\begin{array}{c}\text { Sequence } \\
\text { obtained } \\
\text { (bp) }\end{array}$ & $\begin{array}{c}\text { Blast } \\
\text { Identities } \\
(\%)\end{array}$ & $\begin{array}{l}\text { Identification/ } \\
\text { Accession no. }\end{array}$ \\
\hline 1 & JND-KHGn-29-A & 600 & 582 & 98 & $\begin{array}{c}\text { Bacillus subtilis/ } \\
\text { KU984480 }\end{array}$ \\
\hline 2 & JND-KHGn-29-B & 600 & 547 & 96 & $\begin{array}{c}\text { Bacillus subtilis/ } \\
\text { KU984481 }\end{array}$ \\
\hline 3 & JND-KSGn-30-A & 600 & 587 & 90 & $\begin{array}{c}\text { Bacillus subtilis/ } \\
\text { KU984482 }\end{array}$ \\
\hline 4 & JND-KSGn-30-B & 600 & 578 & 96 & $\begin{array}{c}\text { Bacillus subtilis/ } \\
\text { KU984483 }\end{array}$ \\
\hline 5 & JND-KSGn-30-C & 600 & 643 & 99 & $\begin{array}{c}\text { Bacillus subtilis/ } \\
\text { KU984484 }\end{array}$ \\
\hline 6 & JND-KSGn-30-D & 600 & 609 & 98 & $\begin{array}{c}\text { Bacillus subtilis/ } \\
\text { KU984485 }\end{array}$ \\
\hline 7 & JND-KSGn-30-E & 600 & 619 & 98 & $\begin{array}{c}\text { Bacillus subtilis/ } \\
\text { KU984486 }\end{array}$ \\
\hline 8 & JND-KSGn-30-F & 600 & 535 & 94 & $\begin{array}{c}\text { Bacillus subtilis/ } \\
\text { KU984487 }\end{array}$ \\
\hline 9 & JND-KSGn-30-G & 600 & 579 & 97 & $\begin{array}{c}\text { Bacillus subtilis/ } \\
\text { KU984488 }\end{array}$ \\
\hline 10 & JND-KSGn-30-H & 600 & 623 & 95 & $\begin{array}{c}\text { Bacillus subtilis/ } \\
\text { KU984489 }\end{array}$ \\
\hline 11 & JND-KSGn-30-I & 600 & 604 & 98 & $\begin{array}{c}\text { Bacillus subtilis/ } \\
\text { KU984490 }\end{array}$ \\
\hline
\end{tabular}

$16 \mathrm{~S}$ rRNA B. subtillis gene specific primers pair F: [5'AAGTCGAGCGGACAGATGG 3']

R: [5' CCAGTTTCCAATGACCCTCCCC 3'] 
Fig. 1 In vitro antagonism of bacterial isolates against toxic Aspergillus flavus (JAM-JKBBHA-GG20) on PDA media. A: best antagonist bacterial isolate $B$. subtilis JND-KHGn-29-A on $\mathrm{N}$-agar medium; $\mathbf{B}$ : antagonism after 5 days of inoculation; $\mathbf{C}$ : antagonism after 10 days of inoculation; D: least antagonist bacterial isolate $B$. subtilis JND-KHGn-29-B on N-agar medium;

E: antagonism after 5 days of inoculation; F: antagonism after 10 days of inoculation

\section{B. subtilis JND-KHGn-29-A X A. flavus (JAM-JKB-BHA-GG20)}
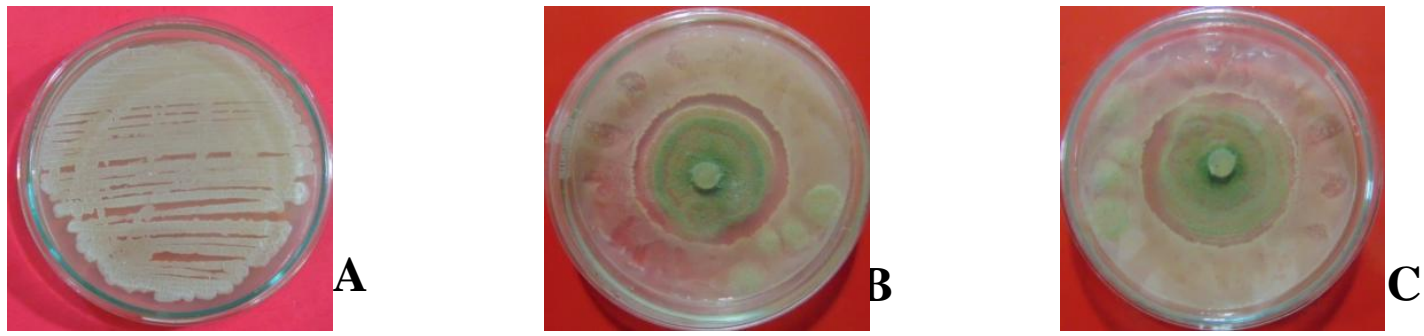

2. B. subtilis JND-KHGn-29-B $X$ A. flavus (JAM-JKB-BHA-GG20)
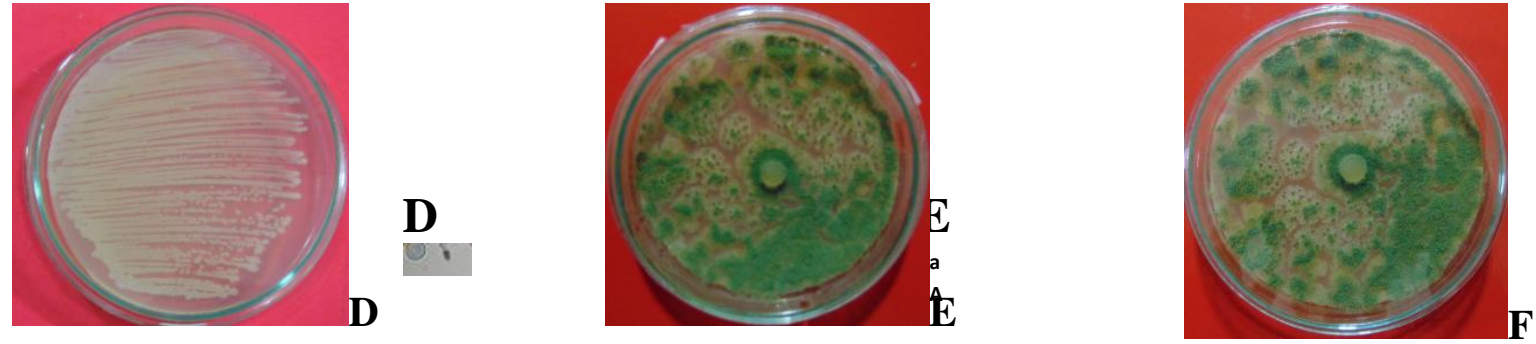

Fig.2 Percent growth inhibition of A. flavus JAM-JKB-BHA-GG20 (Isolate-3) by Bacillus strains at $7 \mathrm{DAI}$

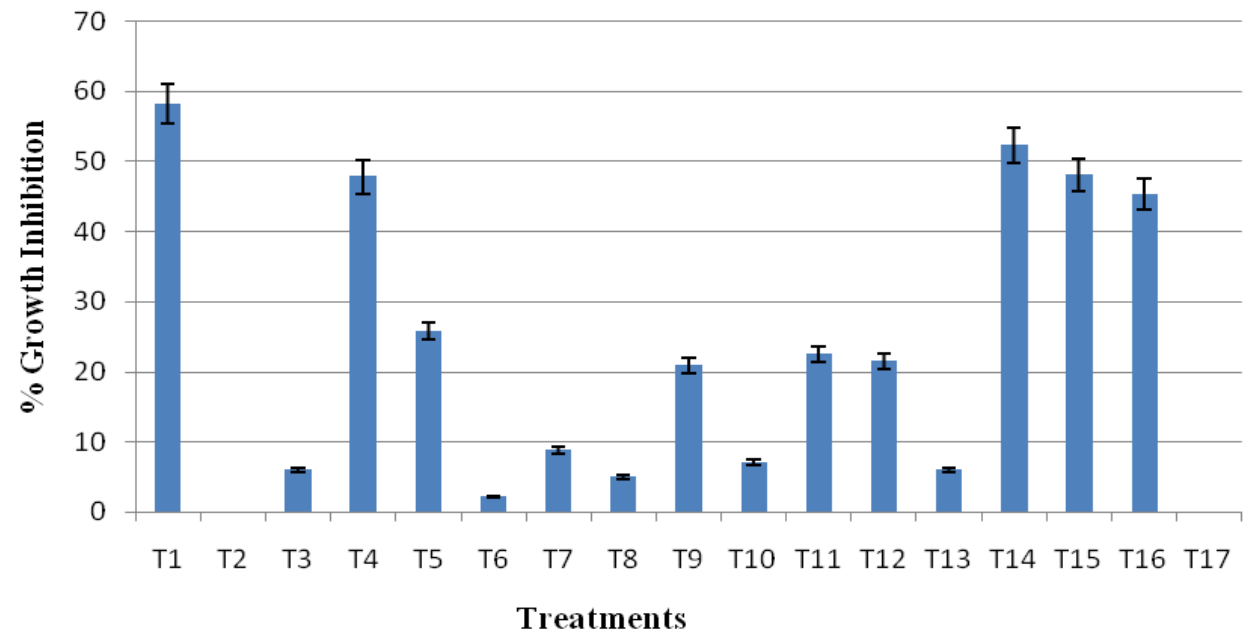


Fig.3 Dendrogram depicting the genetic relationship among the antagonists bacterial isolates based on the RAPD data (1= JND-KHGn-29-A; 2= JND-KHGn-29-B; 3= JND-KSGn-30-A; 4= JND-KSGn-30-B; 5= JND-KSGn-30-C; 6= JND-KSGn-30-D; 7= JND-KSGn-30-E; 8= JND-KSGn-30-F; 9= JND-KSGn-30-G; 10= JND-KSGn-30-H; $11=$ JND-KSGn-30-I; 12= JND-KSGn-30-J; 13= JND-KSGn-30-K; 14= JND-KSGn-30-L; 15= JND-KSCa-22; 16= JND-KSCa-23).

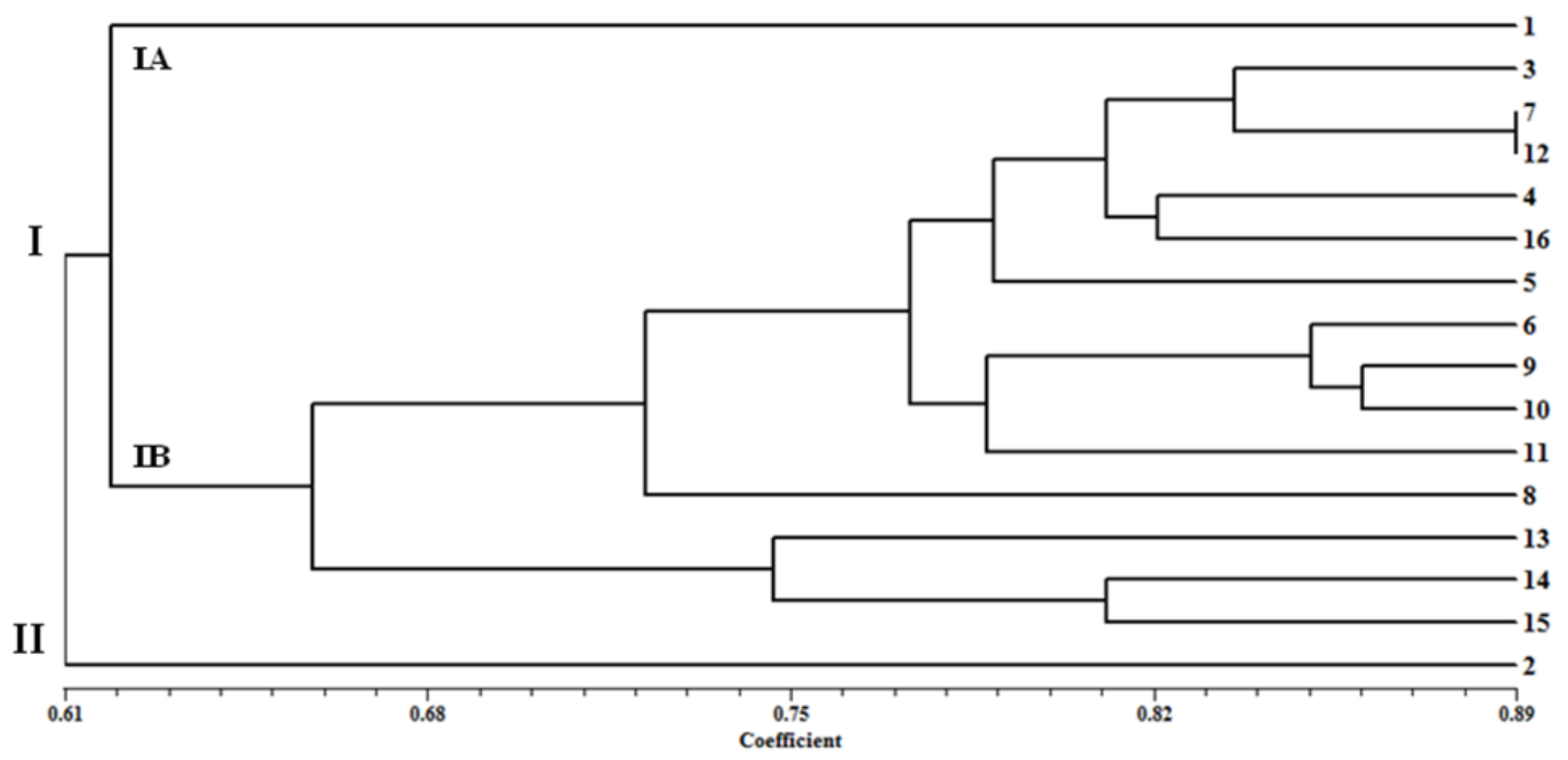


Fig. 4: Dendogram depicting the genetic relationship among the bacterial isolates based on sequencing data corresponding to $16 \mathrm{~S}$ rRNA region

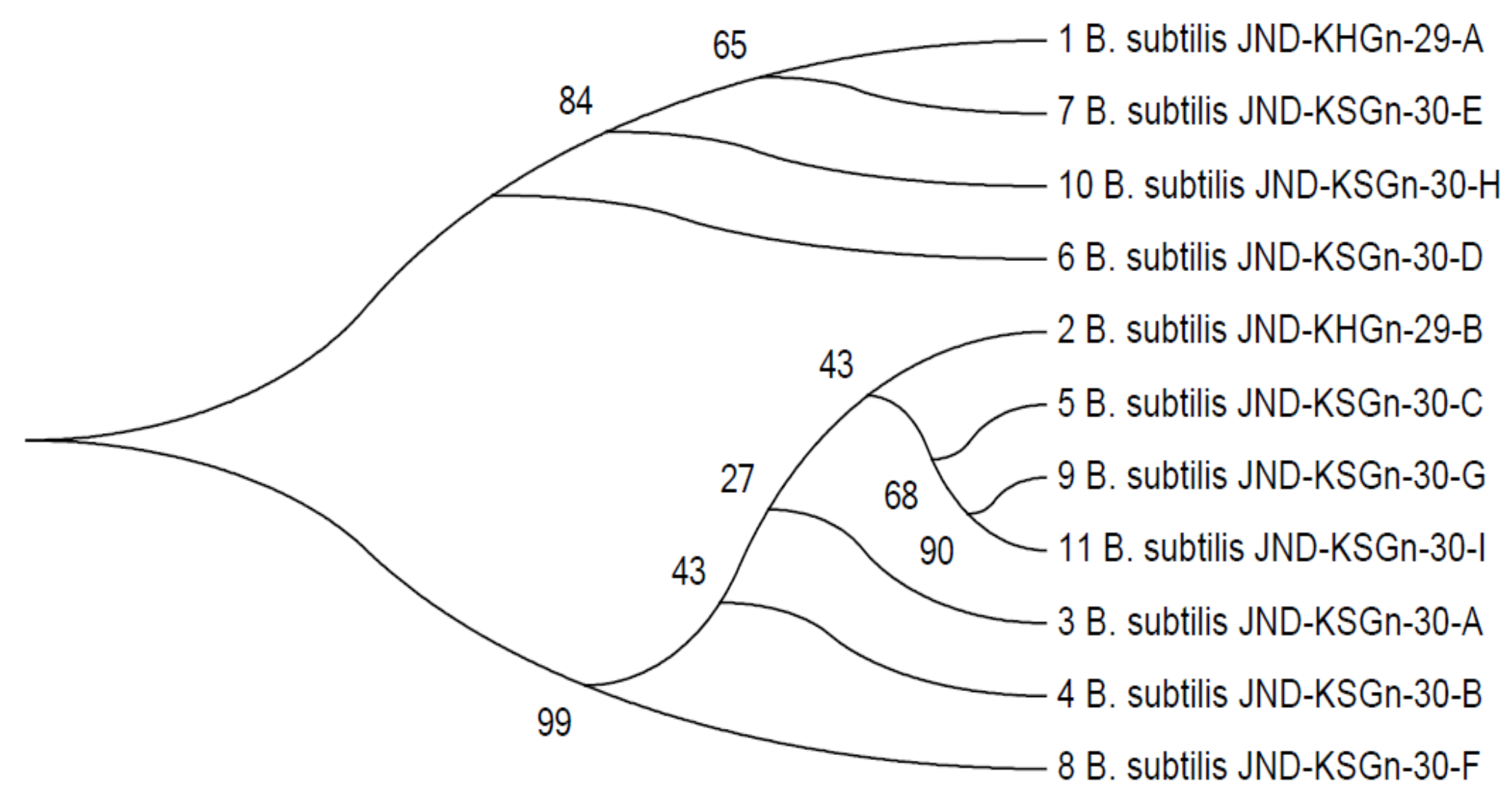


Cluster IA consist of only one bacterial isolate, which was found to be the best antagonist among all 16 bacterial isolates against most toxic and virulent Aspergillus flavus, an identified fungus isolate. Cluster IB grouped most of the bacterial isolates viz. isolate $3,7,12,4,16,5,6,9,10,11,8,13,14$ and 15 . From cluster $\mathrm{B}$, it was observed that isolate JND-KSGn-30-E and isolate JND-KSGn-30-J were closely related at $89 \%$ (Fig. 3). Cluster II consist of only one isolate i.e. isolate JNDKHGn-29-B.

The isolate JND-KHGn-29-B was found least antagonist among all 16 bacterial isolates against most toxic and virulent Aspergillus flavus and it also showed highest genetic dissimilarity with other isolates (Fig. 3). Archana et al. (2007) reported 61\% similarity level after screening 21 isolates with 18 RAPD primers. Prasad (2014) found 56.25\% polymorphism between selected Bacillus cereus species, an enterotoxic pathogenic strains of Bacillus from gut region of local tropical fishes by using 10 primers of the OP series. The results of present study suggest that RAPD primers are effective tool for discremating rhizobacteria in the development of bio-inoculants for disease management in crop plants as the primers were able to distinguish most antagonists and least antagonist bacterium.

\section{Unique RAPD markers associated with antagonistic bacterial isolates}

RAPD markers associated with 16 bacterial isolates were tabulated in Table 5. Out of 38, total 5 primers produce 24 unique bands to identify 16 bacterial isolates. Total 5 primers generate 24 specific unique amplicons viz. the primer OPA- 7 was able to produce 4 unique amplicons within 3 bacterial isolates i.e. 2 unique amplicons in isolate 1 of size $966 \mathrm{bp}$ and $191 \mathrm{bp}$ and isolate 5 of size 493 and 331 and 1 amplicons in isolate 2 of size 875. The primer OPA-18 generates 2 unique amplicons with in two isolates i.e. isolate 1 (1950bp) and 2 (1135). The primer OPK-3 was able to amplify highest 7 amplicons within 16 isolates i.e. 4 amplicons in isolate 1 (1642bp, 671bp, 362bp and 168bp) followed by 2 amplicons in isolate 13 (2308 and 1050) and 1 unique amplicon in isolate $2(305 \mathrm{bp})$. The primer OPG-08 was able to amplify 6 amplicons within 16 isolates i.e. 3 amplicons in isolate 2 (1196bp, 401bp and 281bp) followed by 1 amplicons in each isolate i.e. isolate 1 (650bp), isolate $14(1001 \mathrm{bp})$ and isolate $16(701 \mathrm{bp})$ respectively (Table 5$)$.

Gun-Hee et al. (2009) identified RAPD primers which produced common bands of 0.5 and $0.88 \mathrm{~kb}$ in size with B. subtilis strains. All B. amyloliquefaciens strains generated 1.1 and $1.5 \mathrm{~kb}$ bands together with $0.5 \mathrm{~kb}$ fragment whereas $B$. licheniformis strains produced $1.25,1.70$, and $1.9 \mathrm{~kb}$ bands with an occasional $0.5 \mathrm{~kb}$ band. The $0.5 \mathrm{~kb}$ fragment, the major band for B. subtilis strains, was an internal part of a ytcP gene encoding a hypothetical ABC-type transporter. Fevzi (2001) performed RAPD profiling which revealed the diversity in the Actinomycetes. The number of polymorphic bands observed for each isolates was between 3 and 1 with size ranging from 100 to $2000 \mathrm{bp}$. All the nine isolates characterized on the basis of the RAPD molecular markers produced highly polymorphic patterns. This study help in understanding the difference in bandaing pattern of most antagonists and least antagonist bacterium. No certain reports are avalaable similar to the result of present study which clearly differentiate most antagonist and least antagonist bacterium.

\section{Molecular identification of bacterial isolates using 16S rRNA gene sequencing}

The 16S rRNA is a component of the $30 \mathrm{~S}$ small subunit of prokaryotic ribosomes. The 
genes coding for it are referred to as $16 \mathrm{~S}$ rDNA and are used in reconstructing phylogenies, due to the slow rates of evolution of this region of the gene (Jamal et al., 2013). In present study molecular identification of bacterial strain, PCR was conducted with Bacillus specific universal primers of $16 \mathrm{~S}$ rRNA region (primer pair bsub 5 F [5' AAGTCGAGCGGACAGATGG 3'] - bsub 3 R [5' CCAGTTTCCAATGA CCCTCCCC $\left.3^{\prime}\right]$ ). The specific primers were able to amplify a single amplicon of $600 \mathrm{bp}$ in 11 isolates out of 16 isolates which was further processed for analysis. Therefore, amplified amplicon from Bacillus specific primer set were further taken for sequencing. The obtained sequences were BLAST on NCBI data base. All the BLAST result matches $98 \%$ similarity towards Bacillus subtilis. Therefore, molecular result supports the result obtained from colony characterization. The best antagonist and least antagonist bacterial isolate JND-KHGn-29-A and JND-KHGn-29-B were identified as Bacillus subtilis based on 16S rRNA sequence and both isolates were derived from same healthy rhizospere of groundnut field (Table 6). Therefore, the bacterial isolate JND-KHGn-29-A was identified as Bacillus subtilis. Hall et al. (2003) used the internal transcribed spacers between the $16 \mathrm{~S}$ and the $23 \mathrm{~S}$ ribosomal RNA genes to discriminate species of the 16S rRNA group I of the genus Bacillus by PCR.

\section{Sequenced based phylogenic analysis of 16S rRNA region}

In the present study, determined the $16 \mathrm{~S}$ rRNA gene sequence of 11 isolates form healthy and infected rhizospare of groundnut field. Using BLAST search, it was found that all strains belonged to species Bacillus subtilis. The identities of the 11 Bacillus isolates were determined by comparing them to the available $16 \mathrm{~S}$ rRNA sequences found in
Genbank and with high-scored rRNA sequences in BLAST searches. BLAST similarity scores ranged between $97 \%$ to $100 \%$ (Table 6).

The evolutionary history inferred using the Neighbor-Joining method grouped all 11 analyzed strains in 02 the cluster with a high supported bootstrap. The cluster I grouped 4 isolates (1. JND-KHGn-29-A, 7. JND-KSGn30-E; 10. JND-KSGn-30-H and 6. JNDKSGn-30-D) and cluster II encompassed 7 isolates (2. JND-KHGn-29-B; 5. JND-KSGn30-C; 9. JND-KSGn-30-G; 11. JND-KSGn30-I, 3. JND-KSGn-30-A; 4. JND-KSGn-30B and 8. JND-KSGn-30-F) (Fig. 4).

The 11 Bacillus isolates were clustered based on their antagonist property. The best antagonist bacterial isolate 1 (JND-KHGn-29A) was grouped in cluster I and least antagonist bacterial isolate 2 (JND-KHGn-29B) was grouped in cluster II (Fig. 4).

Jamal et al. (2013) reported 16S sequence size for the 26 isolates, of Bacillus strains grown around Rhazya stricta roots, ranged between 995 to $1233 \mathrm{nt}$, while their counterparts in the Genbank ranged between 1153-1559 nt. Jang et al. (2009) identified potential plant growth promoting (PGP) and antagonistic activities bacterial isolates as Bacillus sp based on 16S rRNA gene sequence after screening seven isolates from rhizosphere of common bean growing at Uttarakhand.

In conclusion, to cope with problems associated with chemical control, an environmentally friendly way of biological control using antagonistic microorganisms is becoming more and more attentive in recent years. The morphological and microscopic characters of bacteria isolates obtained from healthy and infested rhizosphere allows screening for PGPR activity and molecular 
diversity analysis with RAPD markers and $16 \mathrm{~S}$ rRNA sequencing can be employed to distinguish and identify most antagonist and least antagonist bacterium.

\section{References}

Alemu, F. 2013. Isolation of Pseudomonas fluorescens from rhizospheric soil of faba bean and assessment of their Phosphate solubility: in vitro study. Ethiopia. Sch. Acad. J. Biosci., 1: 346351.

Amer, O. E., Mahmoud, M. A., El-Samawaty, A. M. and Sayed, R. M. 2011. Non liquid nitrogen-based method for isolation of DNA from filamentous fungi. Afr. J. Biotechnol., 10: 14371441.

Aneja, K. R. 2003. Experiments in Microbiology, Plant Pathology \& Biotechnology. $4^{\text {th }}$ ed. New Age Int., p.607.

Archana, G., Bhandarkar, M., Dongre, A. B. and Meshram, S. 2007. Genetic Diversity Evaluation of Bacillus isolates using Randomly Amplified Polymorphic DNA molecular marker. Roman. Biotech. Lett., 12(3): 32773286.

Bashan, Y., Holguin G. and de-Bashan L. 2004. Azospirillum-plant relationships: molecular, agricultural, and environmental advances (1997-2003). Can. J. Microbiol., 50:521-577.

Chapon, A., Morgane, B., Delphine, R., Laurie, D., Guillerm, A. and Sarniguet, A. 2002. Direct and specific assessment of colonisation of wheat rhizoplane by Pseudomonas fluorescens Pf29A. Euro. J. Pl. Patho., 109: 61-70.

Clark V.L. and Bavoil P.M. 1994. Bacterial pathogenesis. In. Abelson JN and Simon MI (Editors). Methods in
Enzymology. San Diego, Academic Press. 235 p. 324-357.

Fevzi, B. 2001. Random Amplified Polymorphic DNA (RAPD) Markers. Turk J. Biol., 25: 185-196.

Fisher, R. A. and Yates, N. D. 1948. Statistical methods for research workers. Oliver and Boyd, Edinburg, London. 12th ed-Bio. Monograph and Manuals., 5: 130-131.

Glick R. and Bernard. 2012. Plant GrowthPromoting Bacteria: Mechanisms and Applications. Hindawi Publishing Corporation Scientifica, Volume 2012, Article ID 963401, 15 pages.

Gun-Hee K., Hwang, A. L., Jae-Young, P., Jong, S. K., Jinkyu, L., Cheon, S. P., Dae, Y. K., Yong, S. K. and Jeong, H. K. 2009. Development of a RAPDPCR method for identification of Bacillus species isolated from Cheonggukjang. Internat. J. Food Microbiol., 129: 282-287.

Hall, L., Doerr, K. A., Wohlfiel, S. L. and Roberts, G. D. 2003. Evaluation of the MicroSeq System for identification of Mycobacteria by $16 \mathrm{~S}$ ribosomal DNA sequencing and its integrations into a routine clinical mycobacteriology laboratory. J. Clin. Microbiol., 41: 1447-1453.

Hayat R., Ali S., Siddique M. T. and Chatha T. H. 2008a. Biological nitrogen fixation of summer legumes and their residual effects on subsequent rainfed wheat yield. Pakistan J. Bot., 40(2): 711-722.

Hayat R., Ali S., Ijaz S. S., Chatha T. H. and Siddique M.T. 2008b. Estimation of N2-fixation of mung bean and mash bean through xylem uriedetechnique under rainfed conditions. Pakistan J. Bot. 40(2):723-734.

Jamal S. M., Sabir, S. E. M., Abo-Aba, Ayman S., Refaei M. H., Ahmed, B. and Nabeeh, A. B. 2013. Isolation, 
identification and comparative analysis of 16S rRNA of Bacillus subtilis grown around Rhazya stricta roots. Life Sci. J., 10(12): 121-124.

Jang, S. K., Wen, Z., and Pei Y. Q. 2009. Discovery of marine Bacillus species by $16 \mathrm{~S}$ rRNA and rpoB comparisons and their usefulness for species identification. J. Microbiol. Meth., 77:48-57.

Jeyaram, K., Singh M. W., Premarani, T., Devi, A.R., Chanu, K.S., Talukdar, N.C., Singh, M.R., 2008. Molecular identification of dominant microflora associated with 'Hawaijar'-a traditional fermented soybean (Glycine $\max$ L.) food of Manipur, India. Int. J. of Food Microbiol., 122: 259-268.

Joseph, B., Patra, R. R., and Lawrence, R. 2007. Characterization of plant growth promoting Rhizobacteria associated with chickpea (Cicer arietinum L). Int. J. of Plant Production., 1(2): 141-152.

Kennedy, I. R., Choudary, A.I.M.A. and Kecskes, M.L. 2004. Non Symbiotic bacterial diazotrophs in a crop farming systems can their potential for plant growth promotion to be better exploited? Soil Biol. Biochem., 36(8): 1229-1244.

Kim, J. S., Kuk, E., Yu, K. N., Kim, J. H. and Park, S. J. 2007. Antimicrobial effects of silver nanoparticles, Nanomed., 3: 95-101.

Levine, S.M., Tang, Y.W. and Pei, Z.H. 2005. Recent advances in the rapid detection of Bacillus anthracis. Rev. Med. Microbiol., 16:125-133.

Ligon, J. M., Hill, D. S., Hammer, P. E., Torkewitw, N. R., Hofman, D., Kempt, H. J. and van Pee, K.H. 2000. Natural products with antifungal activity from Pseudomonas biocontrol bacteria. Pest Manag. Sci., 56: 688695.
Martinez, M. A., Delgado, O. D., Breccia, J. D., Baigori, M. D. and Sineriz, F. 2002. Revision of the taxonomic position of the xylanolytic Bacillus sp. MIR32 reidentified as Bacillus halodurans and plasmid-mediated transformation of B. halodurans. Extremophiles., 6: 391-395.

Meyer, J. M. and Stintzi, A. 1998. Iron metabolism and siderophore production in Pseudomonas and related species. In Montie, ed. Pseudomonas. New York: Plenum Press, pp. 201-243.

Nakano, S., Peberdy, J. F., and Lumyong, S. 1998. Indole-3-acetic acid production by Streptomyces sp.isolated from some Thai medicinal plant rhizosphere soils. Eur. Asia J. Biosci. 4: 31-34.

Noori, M. S. S. and Saud, H. M. 2012. Potential Plant Growth-Promoting Activity of Pseudomonas sp. Isolated from Paddy Soil in Malaysia as Biocontrol Agent. J. Plant Pathol. Microbiol., 3:120.

Owen, A. and Zlor R. 2001. Effect of cyanogenic rhizobacteria on the growth of velvetleaf (Abutilon theophrasti) and Corn (Zea mays) in autoclaved soil and the influence of supplemented glycine. Soil Biochem., 33: 801-809.

Pidello, A. 2003. The effect of Pseudomonas fluorescens strains varying in pyoverdine production on the soil redox status. Plant Soil., 253: 373379.

Prasad, M. P. 2014. Molecular characterization of Enterotoxigenic Bacillus cereus species isolated from tropical marine fishes using RAPD markers. Int. J. Pure App. Biosci., 2(4): 189-195.

Raaijmakers, J. M., Vlami, M. and de Souza, J. T. 2002. Antibiotic production by bacterial biocontrol agents. Antonie 
Van Leeuwenhoek., 81: 537-547.

Raval, A. A. and Desai, P. B. 2012. Rhizobacteria from rhizosphere of sunflower (Helianthus annuus L.) and their effect on plant growth. Res. J. Rec. Sci., 1(6): 58-61.

Ravikumar, P., Hutson, R. A. and Naresh, K. G. 2002. Involvement of a phosphate starvation inducible glucose dehydrogenase in soil phosphate solubilization by Enterobacter asburiae. FEMS Microbiol. Lett., 171: 223-229.

Reddy, B. P., Reddy, K. R. N., Rao, M. S. and Rao, K. S. 2008. Efficacy of antimicrobial metabolites of Pseudomonas fluorescens against rice fungal pathogens. Current Trends Biotechnol. Pharm., 2: 178-182.

Ronimus, R.S., Parker, L.E. and Morgan,
H.W. 1997. The utilization of RAPDPCR for identifying thermophilic and mesophilic Bacillus species. FEMS Microbiol. Lett., 147:75-79.

Torriani, S., Clementi, F., Vancanneyt, M., Hoste, B., Dellaglio, F., Kersters, K., 2001. Differentiation of Lactobacillus plantarum, $L$. pentosus and $L$. paraplantarum species by RAPD-PCR and AFLP. Syst, Appl. Microbiol., 24, 554-560.

Zarrin, F., Saleemi, M., Zia, M., Sultan, T., Aslam, M., Rehman, R. and Chaudhary, M. F. 2009. Antifungal activity of plant growth-promoting rhizobacteria isolates against Rhizoctonia solani in wheat. Afri. J. of Biotech., 8: 219-225.

\section{How to cite this article:}

Bharose, A.A., H.P. Gajera, Darshna G. Hirpara, V.H. Kachhadia and Golakiya, B.A. 2017. Molecular Identification and Characterization of Bacillus Antagonist to Inhibit aflatoxigenic Aspergillus flavus. Int.J.Curr.Microbiol.App.Sci. 6(3): 2466-2484. doi: https://doi.org/10.20546/ijcmas.2017.603.280 